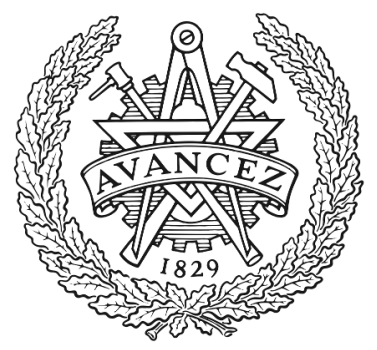

CHALMERS

UNIVERSITY OF TECHNOLOGY

\title{
Simultaneous and proportional estimation of hand kinematics from EMG during mirrored movements at multiple degrees-of-freedom
}

Downloaded from: https://research.chalmers.se, 2023-04-26 01:43 UTC

Citation for the original published paper (version of record):

Muceli, S., Farina, D. (2012). Simultaneous and proportional estimation of hand kinematics from EMG during mirrored movements

at multiple degrees-of-freedom. IEEE Transactions on Neural Systems and Rehabilitation

Engineering, 20(3): 371-378. http://dx.doi.org/10.1109/TNSRE.2011.2178039

N.B. When citing this work, cite the original published paper.

(C2012 IEEE. Personal use of this material is permitted.

However, permission to reprint/republish this material for advertising or promotional purposes 


\title{
Simultaneous and Proportional Estimation of Hand Kinematics from EMG during Mirrored Movements at Multiple Degrees of Freedom
}

\author{
Silvia Muceli, Student Member, IEEE, Dario Farina*, Senior Member, IEEE
}

\begin{abstract}
This paper proposes and tests on able-bodied subjects a control strategy that can be practically applied in unilateral transradial amputees for simultaneous and proportional control of multiple degrees of freedom (DOFs). We used artificial neural networks to estimate kinematics of the complex wrist/hand from high-density surface EMG signals of the contralateral limb during mirrored bilateral movements in free space. The movements tested involved the concurrent activation of wrist flexion/extension, radial/ulnar deviation, forearm pronation/supination and hand closing. The accuracy in estimation was in the range $79-88 \%\left(r^{2}\right.$ index) for the four DOFs in six able-bodied subjects. Moreover, the estimation of the pronation/supination angle (wrist rotation) was influenced by the reduction in the number of EMG channels used for the estimation to a greater extent than the other DOFs. In conclusion, the proposed method and set-up provide a viable means for proportional and simultaneous control of multiple DOFs for hand prostheses.
\end{abstract}

Index Terms-Electromyography, degrees of freedom, kinematics, prosthetic control

\section{INTRODUCTION}

$\mathrm{R}$ esearch in myoelectric control has produced active prosthetic devices with many degrees of freedom (DOFs), paralleled by EMG-based control strategies. Nevertheless, these advances did not proceed at the same speed. Advances in mechatronics have yielded multi-fingered prosthetic hands capable of mimicking the functions that the human counterpart provides (Cyberhand [1], Touch Bionics's i-LIMB [2]). On the other hand, most commercial upper limb prostheses (e.g., Otto Bock hands [3]) are still one DOF grippers because of limitations in controlling more complex systems with current EMG processing methods. Many attempts have been carried out to increase the number of functions to be controlled. In research settings, classification algorithms have been proposed for decoding multiple DOFs, e.g. forearm pronation and hand

Manuscript received June 24, 2011. This work was supported by Regione Autonoma Sardegna through a Master\&Back doctoral fellowship.

Asterisk indicates corresponding author.

The authors are with the Center for Sensory-Motor Interaction, Department of Health Science and Technology, Aalborg University, Aalborg DK-9220, Denmark and with the Department of Neurorehabilitation Engineering, Bernstein Center for Computational Neuroscience, Georg-August University of Göttingen, Göttingen, Germany (e-mail: silvia.muceli@bccn.unigoettingen.de, dario.farina@bccn.uni-goettingen.de). closing $[4,5]$. However, these approaches are limited to controlled laboratory conditions.

Current myoelectric control is not adequate for actuating all the functions offered by the prosthetic technology available nowadays and thus to replicate the motor capability of the amputated hand. For example, pattern recognition based myoelectric control systems employ a sequential strategy, where only one function of the prosthetic device can be active at a time. Conversely, natural limb movements consist in the continuous and simultaneous activation of multiple DOFs, which is required even by the simplest daily life activities, e.g. pouring water from a bottle. The absence of combined functions forces the users of myoelectric prostheses to actuate unnatural strategies to achieve movements that normallylimbed individuals accomplish effortlessly. In turn, this implies a high level of cognitive effort put into the control of the prosthesis and consequently a low degree of acceptance. Cosmetic prostheses are thus often preferred to active prostheses [6].

The simultaneous control of multiple DOFs movements was analyzed by Jiang et al. [7] who proposed a semi-unsupervised algorithm capable of providing a linear mapping between multichannel EMG amplitude and force produced during wrist contractions involving flexion/extension, radial/ulnar deviation, and the combination of both. Due to its semiunsupervised nature, the algorithm does not require recording of the force signals, which is important in practical applications since forces cannot be recorded from amputees. However, this approach failed when applied to wrist pronation/supination. This effect could be mitigated by using an artificial neural network (ANN) able to capture the nonlinearity of the system. The drawback of using an ANN is that its training requires recordings of the force exerted from the hand ipsilateral to the arm from which the EMG is recorded. This is not possible in amputees. Nielsen et al. [8] faced this issue by proving that the surface EMG recorded from one upper limb can be used to estimate the force produced by the contralateral limb in the case of bilateral, symmetric contractions. That study was however still limited to only two DOFs. The difficulties in estimating pronation/supination forces were also related to force translation across the three DOFs introduced by the experimental apparatus used to gather force [7], which restrained the subject's hand. 
The present study aims at overcoming the above limitations by presenting a method for the continuous extraction of control information during movements which involve simultaneous activation of the three DOFs of the wrist, in addition to hand closing. We concurrently recorded the kinematics of both hands and the surface EMG from forearm muscles (unilaterally) while the subjects performed bilateral, mirrored movements, and we estimated the kinematics of the contralateral hand from the EMG using ANNs. Since in this study the subjects executed dynamic tasks in the free space and with a greater number of DOFs, we propose the use of high-density recordings [9] in an attempt to increase the information extracted from the surface EMG. In myoelectric control, not more than 8 bipolar channels are usually employed as the source of control $[4,8,10,11]$. This suffices in decoding movements which are controlled by superficial muscles whose activity is easily detected at the surface of the skin (e.g., flexion/extension). However, anatomical factors hamper the detection of muscles responsible for pronation/supination. Precisely, the pronator teres is partly covered by the bicipital aponeurosis and the supinator muscle is deep [12]. We hypothesized that high-density surface EMG recordings [9] would allow to better exploiting the spatial information across the muscles compared with bipolar recordings routinely used in myoelectric prosthetic control studies $[4,7,8,10,11]$. As in other studies $[13,14]$, we mapped EMG to kinematics rather than force while subjects performed movements in free space.

This paper describes a new strategy to estimate the kinematics of the contralateral hand from the EMG of forearm muscles during mirrored, bilateral movements which involve the combined articulation of four DOFs of the complex wristhand in free space.

\section{METHODS}

\section{A. Subjects}

Similar to most recent studies that proposed novel strategies for myoelectric control [5, 15-17], we analyzed our method in normally-limbed subjects, which is a necessary basis for future testing in amputees. Six normally-limbed subjects (age, $28.3 \pm$ $6.4 \mathrm{yrs}$ ) participated to the experiment, after providing written informed consent. The procedures were approved by the local ethic committee.

\section{B. Procedure}

The subject was standing with the arms along the body in a resting semipronated position (palm facing inward). The protocol included 7 single DOF movements and 14 combined movements, as listed in Table I. The selection of movement combinations was based on functionally relevant tasks.

TABLE I

MOVEMENTS INCLUDED IN THE PROTOCOL. THE TASKS WERE PERFORMED BY THE SUBJECTS IN BLOCKS (BLOCK INDEX) AND RANDOMIZED WITHIN EACH BLOCK (MOVEMENT INDEX). THE ORDER OF THE BLOCKS WAS ALSO RANDOMIZED.

\begin{tabular}{lll}
\hline \hline $\begin{array}{l}\text { Block } \\
\text { index }\end{array}$ & $\begin{array}{l}\text { Movem } \\
\text { index }\end{array}$ & \\
\hline & 1 & Rest \\
1 & 2 & Wrist flexion \\
1 & 3 & Wrist extension \\
1 & 4 & Radial deviation \\
1 & 5 & Ulnar deviation \\
2 & 6 & Forearm pronation \\
2 & 7 & Forearm supination \\
2 & 8 & Hand closing \\
3 & 9 & Forearm pronation \& Wrist flexion \\
3 & 10 & Forearm pronation \& Wrist extension \\
3 & 11 & Forearm pronation \& Radial deviation \\
3 & 12 & Forearm pronation \& Ulnar deviation \\
4 & 13 & Forearm supination \& Wrist flexion \\
4 & 14 & Forearm supination \& Wrist extension \\
4 & 15 & Forearm supination \& Radial deviation \\
4 & 16 & Forearm supination \& Ulnar deviation \\
5 & 17 & Hand closing \& Wrist flexion \\
5 & 18 & Hand closing \& Wrist extension \\
5 & 19 & Hand closing \& Radial deviation \\
5 & 20 & Hand closing \& Ulnar deviation \\
5 & 21 & Hand closing \& Forearm pronation \\
5 & 22 & Hand closing \& Forearm supination \\
\hline \hline
\end{tabular}

The subject was instructed to perform a series of mirrored, bilateral, dynamic contractions starting from the resting position. The movements to be reproduced were displayed on a computer screen located in front of the subject. Each movement had a duration of $6 \mathrm{~s}$, with $1 \mathrm{~s}$ of absence of activity (pre-movement phase), approximately $1 \mathrm{~s}$ of movement from the initial to the final position, maintenance of the final position for approximately $1 \mathrm{~s}$, approximately $1 \mathrm{~s}$ of movement from the final to the initial position, and absence of activity (post-movement phase) for the remaining time interval. Following a period of familiarization with the protocol, the subjects performed each of the 22 movements listed in Table I 4 times, divided in 5 blocks of trials to minimize fatigue and the need of concentration. At least 5 minutes of rest was provided between the blocks of trials and extended under the discretion of the subjects.

Surface EMG signals were detected from forearm muscles of the right limb and hand kinematics of both limbs was concurrently recorded during the mirrored bilateral movements.

\section{EMG Recordings}

Surface EMG signals were recorded from forearm muscles using two semi-disposable adhesive grids of 64 electrodes each arranged in 5 rows and 13 columns with $8 \mathrm{~mm}$ of interelectrode distance (LISiN-OT Bioelettronica, Torino, Italy), placed around the circumference of the right forearm at a distance from the elbow of one third of the elbow-wrist distance (Fig. 1). Prior to electrode placement the skin was shaved, if necessary, and lightly abraded. The EMG signals were amplified with a gain of 2000 (EMG-USB, LISiN - OT Bioelettronica, Torino, Italy), band-pass filtered (8th order Bessel filter, bandwidth 10-750 Hz), sampled at $2048 \mathrm{~Hz}$, and $\mathrm{A} / \mathrm{D}$ converted on 12 bits. A reference electrode was placed at the wrist of the right arm. 


\section{Kinematics}

Two sets of seven reflective ball shaped markers (12-mm diameter) were symmetrically placed on the two arms of the subject (Fig. 1). Two markers were positioned parallel to the medial (MEP) and lateral (LEP) epicondyles of the humerus through a rigid plastic fixture. The other five markers were placed in correspondence of the styloid processes of radius (STR) and ulna (STU), the radial head of the second metacarpal bone (RMC), the ulnar head of the fifth metacarpal bone (UMC) [18], the radial side of the joint between the proximal phalanx and the middle phalanx of the index finger (FIN). The positions of the markers were tracked with a motion analysis system (Qualisys Track Manager, Qualisys $\mathrm{AB}$, Gothenburg, Sweden) with 8 infrared digital video cameras (ProReflex MCU, Qualisys AB, Gothenburg, Sweden). The kinematics data were recorded with a sampling frequency of $256 \mathrm{~Hz}$.

\section{E. EMG Processing}

The EMG signals were off-line band-pass filtered (4th order zero-lag Butterworth digital filter, pass-band $20-400 \mathrm{~Hz}$ ) to attenuate DC offset, motion artifacts, and high frequency noise [19]. Two matrices provided 102 bipolar channels in the direction of the muscle fibers. Signals were visually inspected before processing and signals corresponding to missing contacts were eliminated. The number of bipolar channels discarded was within the range 5-16. Therefore, hereafter, we refer to all the channels to indicate the full number of available channels after discarding the bad contacts, which corresponded to channels in the range 86-97, depending on the subject. The filtered signals were full-wave rectified and lowpass filtered (2th order zero-lag Butterworth digital filter, cutoff frequency $16 \mathrm{~Hz}$ ) to obtain the muscle activity envelopes and synchronized with the kinematics signals at a common sampling rate of $32 \mathrm{~Hz}$.

\section{F. Kinematics Data Processing}

Movements were quantified in terms of angular displacement with respect to the resting position, according to [18]. Briefly, a reference coordinate system for the hand was defined as follows (ELB is defined as the midpoint between MEP and LEP) (Fig.1):

- origin (O): midpoint between STR and STU,

- z-axis: line O-ELB, pointing to ELB,

- y-axis: line perpendicular to the plane STR-STU-ELB and passing through $\mathrm{O}$, pointing anteriorly,

- $\mathrm{x}$-axis: cross product by the right hand rule.

The flexion/extension (radial/ulnar deviation) angle was calculated as angular displacement of the projection of the vector O-HAN, HAN being the midpoint between RMC and $\mathrm{UMC}$, on the plane $\mathrm{y}-\mathrm{z}(\mathrm{x}-\mathrm{z})$. The pronation/supination angle was estimated from the projections of the lines STR-STU and MEP-LEP on a plane perpendicular to the line O-ELB.

Since the fingers move in agreement during the opening/closing, the movement of the index finger was considered as indicative of the global closing movement. Therefore, the opening/closing angle was calculated as the angle between the planes O-UMC-RMC and UMC-RMC-FIN.

Positive values corresponded to flexion, radial deviation, pronation and closing. The angles were low-pass filtered by a second-order dual-pass Butterworth filter at a cutoff frequency of $1 \mathrm{~Hz}$ (see next Subsection).

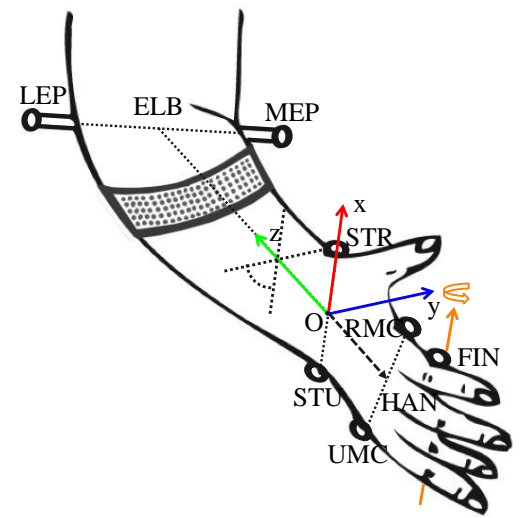

Fig. 1. Marker position and hand reference system. LEP: lateral epicondyle; MEP: medial epicondyle; ELB: elbow; STR: styloid process of radius; STU: styloid process of ulna; RMC: radial metacarpal bone; UMC: ulnar metacarpal bone; FIN: finger; HAN: hand; O-X-y-z: hand reference system.

\section{G. Estimation of Hand Kinematics from the EMG}

ANNs were used to learn the association between EMG and hand kinematics. The EMG signal is known to be related to the force produced by the muscle [20]. Ultimately, the forces developed by all the muscles acting on a joint determine the position of that joint. The setup we chose ensures a welldefined relation between position and force since each position requires a specific force to overcome gravity. We thus assume that the ANNs can learn the associations EMG-force and force-position in order to estimate position from EMG. Static ANNs were used because for this application they perform as well as time-delayed ANNs in spite of the simpler architecture [18]. Eight multilayer perceptrons (MLPs) were used to estimate the flexion/extension, radial/ulnar deviation, pronation/supination and opening/closing angles of the ipsi and contralateral hand. We used different MLPs for different angles with the aim of obtaining an accurate estimation for each DOF. Each MLP had the bipolar EMG channels envelopes (or a selection/projection of them, as specified in Subsection $\mathrm{H}$ ) as input and one of the angles as target. All the MLPs had one hidden layer with a number of neurons varying between 1 and 10 during training while the estimation error was monitored. The neurons in the hidden and output layers had a sigmoid and a linear activation function, respectively. The MLPs were trained using the Levenberg-Marquardt backpropagation algorithm [21]. The network output was low-pass filtered at $1 \mathrm{~Hz}$ to match the frequency content of the network target (see Kinematics Data Processing). Such a cut-off frequency may appear low. Preliminary tests were done limiting the kinematics bandwidth to $6 \mathrm{~Hz}$ [22]. However, from the signals collected in this study, the network target had the majority of the power in the frequency band below $1 \mathrm{~Hz}$. Therefore, we further low-pass filtered kinematics data at 1 $\mathrm{Hz}$.

A four-fold cross validation procedure was used to evaluate 
the MLP performance, where 3 out of the 4 repetitions of each movement were used for training/validation (70\%/30\% respectively), whereas the fourth was used to test the behavior of the MLP in presence of novel inputs. Previous studies [7, 8] provided an indication of the global quality of the estimation (i.e. without differentiating among the different DOFs) through the multivariate coefficient of determination [23], whereas we are interested in assessing how accurately each single DOF was estimated. Therefore, the performance of the MLP in the estimation of each angle was evaluated through the coefficient of determination $r^{2}$ (the percent variability in the actual angular values explained by the estimated values) [18] and the mean relative error $r E$ (the magnitude of the difference between the measured and the estimated value, divided by the range of motion).

For future applications in unilateral amputees, the kinematics can be extracted for training from the limb contralateral to the one used to gather EMG. Nonetheless, in this study the kinematics was recorded bilaterally to verify afterwards that movements of the two hands were mirrored and synchronized (using the peak of the normalized crosscorrelation function).

\section{H. Feature Analysis}

Since the inter-electrode distance was $8 \mathrm{~mm}$, signals from adjacent channels were highly correlated. Therefore, we investigated if the dimensionality of the inputs needed for learning might be reduced via feature selection/projection.

Channel selection is graphically represented in Fig. 2 for the different tests made. Seven and 14 equally spaced channels around the forearm were selected in some tests (Fig. 2A,B). The possibility of using linear arrays of electrodes rather than matrices was also investigated through the selection of 26 channels, corresponding to a row of the matrices (Fig. 2C).

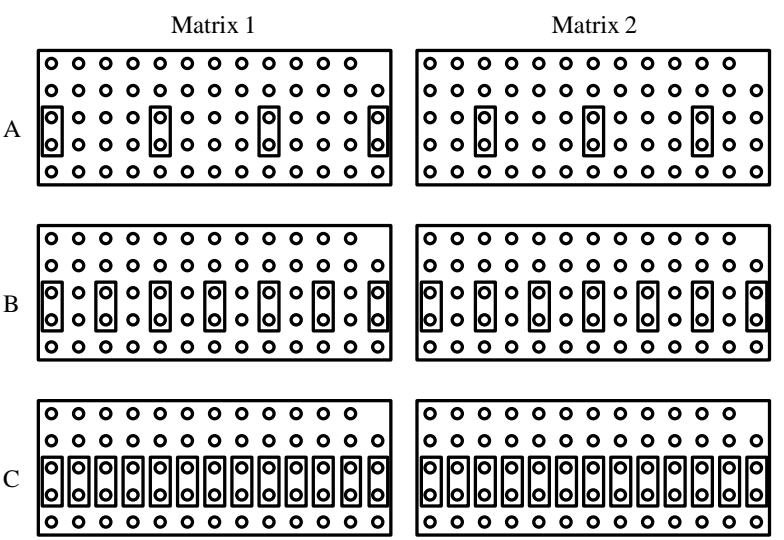

Fig. 2. Channel selection. Seven (A), 14 (B) and 26 (C) bipolar channels along the muscle fiber direction were selected as input for the MLPs in different tests. The results for these channel selections were compared to the results obtained using all bipolar channels.

In addition to channel selection, the reduction in the feature space was also investigated by applying Principal Component Analysis (PCA) [24] to the envelopes of the original channels. Fourteen principal components sufficed to explain $>95 \%$ of the EMG envelopes variance for all subjects (see RESULTS).
Therefore, the first 7 and 14 components were retained for comparison.

The MLPs training was repeated using the channels subset/principal components as input. ANOVA followed by the post-hoc Student Newman-Keuls test was applied to investigate the effect of channel selection/projection on the resulting performance. Separate ANOVAs were performed for the four angles. ANOVA was also applied to investigate if performance from the ipsi and contralateral limbs differed. Statistical significance was set at $\mathrm{P}<0.05$.

\section{RESULTS}

The number of neurons in the hidden layer influenced the performance, however extensive preliminary data analysis (results not shown for clarity) indicated that when using more than 6 neurons the performance did not improve further, irrespective of the number of features provided as input. Therefore, 6 neurons were used in the hidden layer of each MLP in the subsequent results.

The subjects performed symmetric movements in concert with both limbs. The peak of the normalized cross-correlation function between corresponding angles at the two sides was $0.83 \pm 0.16$ (average across the four DOFs and the 6 subjects). The estimation performance for the ipsi and contralateral limbs were similar for all the DOFs and all input features (both in terms of the coefficient of determination and the mean relative error), with the only exception of the estimation of the pronation/supination angle. For this angle, when using all channels as input, the ipsilateral estimation was slightly but significantly better than the contralateral estimation $\left(r^{2}, 86.5 \pm\right.$ $3.2 \%$ vs $83.5 \pm 3.4 \%$; $r E, 3.7 \pm 0.5 \%$ vs $4.2 \pm 0.6 \%$; $\mathrm{P}<0.05)$. Hereafter, we detail the results for the estimation of the contralateral hand kinematics only, because this is the only viable training solution for amputees and thus the most relevant.

Fig. 3 displays representative results from one subject (contralateral case) to illustrate the quality of angular position estimation by the MLPs when using all the channels. The estimation is of high quality in this example. The protocol prescribed the activation of a single DOF or two DOFs at a time. However, the angle traces show that the movements that the subjects were requested to reproduce implied the activation of other DOFs as well (see Fig. 3), due to the fact that muscles controlling the hand operate in a synergistic fashion [7]. It is worth noting that the estimation had good accuracy even during the concurrent involvement of the 4 DOFs (Fig. 3).

Group data are represented in Fig. 4, which shows the average coefficient of determination (A) and the mean relative error (B) between the measured and the estimated angular displacement for the four DOFs for the contralateral hand when varying the input features. 


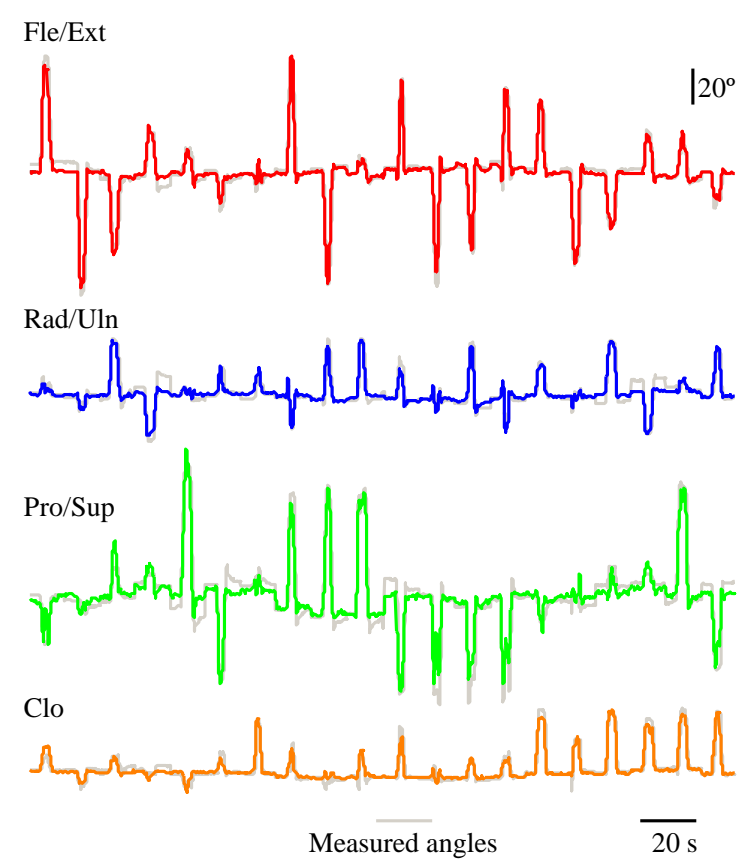

Fig. 3. Measured and estimated angular displacements for one representative subject during combined activation of the four DOFs. In this example, the coefficient of determination and the mean relative error were $93.8 \%(1.9 \%)$ for flexion/extension, $86.3 \%$ (3.5\%) for radial/ulnar deviation, $89.8 \%(3.3 \%)$ for pronation/supination and $88.9 \%$ (3.1\%) for closing. Estimates are obtained for the contralateral hand.

The results obtained with $r^{2}$ and $r E$ were consistent, although differences in the estimation quality were more evident when using the coefficient of determination. The values of $r^{2}$ and $r E$ differed depending on the inputs $(\mathrm{P}<<0.001$ for each of the angles), with superior performance when using all channels than in the other conditions $(\mathrm{P}<0.01$ in all cases, not indicated in Fig. 4 for clarity). The $r^{2}$ values were $88.5 \pm 4.4 \%, 79.3 \pm 3.9 \%, 83.5 \pm 3.4 \%$, and $83.8 \pm 3.8 \%$ for flexion/extension, radial/ulnar deviation, pronation/supination and closing, respectively. The worst performance corresponded to the configuration of 7 equally spaced channels around the circumference of the forearm $(77.5 \pm 8.0 \%, 64.8 \pm 8.0 \%, 43.7 \pm 6.7 \%, 68.7 \pm 6.0 \%)$. Interestingly, $r^{2}$ was halved for pronation/supination with this configuration with respect to the full number of channels. Fourteen channels yielded better performance than 7. Twentysix channels further improved the quality of the estimation but only for the pronation/supination angle. The linear array configuration (Fig. 2C) yielded a coefficient of determination of $83.5 \pm 6.7 \%, 72.2 \pm 5.8 \%, 67.4 \pm 7.9 \%$, and $78.4 \pm 4.0 \%$ for the four angles. The reduction in the $r^{2}$ with respect to the matrix configuration was about $15 \%$ for pronation/supination, and only about 5\% for the other DOFs. Despite the reduction in number of channels, the performance can still be considered generally acceptable, as confirmed by the fact that the mean relative error was inferior to $6 \%$ with such a reduced configuration.

Seven (14) components accounted for $92.6 \pm 2.5 \%$ (96.7 \pm $0.8 \%)$ ) of the EMG envelopes variance. Fourteen principal components outperformed 7 components regardless of the DOF. The number of channels being equal, channel projection resulted superior to channel selection only in case of flexion/extension and pronation/supination.

\section{DISCUSSION}

This paper has proposed the estimation of the contralateral hand kinematics during movements which involve combined activation of four DOFs of the hand in free space from highdensity surface EMG.

\section{A. Simultaneous Control of Multiple DOFs}

The proposed method showed performance comparable or superior to those obtained in other studies on simultaneous control of multiple DOFs $[7,8,11]$, although validated in a more complex scenario. First of all, our control scheme comprises the hand closing, whereas previous studies on simultaneous activation of multiple DOFs [7, 8, 11, 18] only considered the wrist as joint of interest. Hand closing, however, is a fundamental function in prosthesis control, included in all commercial devices. Moreover, the protocol followed in $[7,8,11]$ requested to restraint the subject's hand, whereas we focused on dynamic movements in free space, which are inherently more variable and closer to real scenarios. The fact that in current research, training and test data are often acquired with the arm in a constrained setting maybe one of the cause of the degradation of performance when the prosthesis is used in everyday life [25]. It should be pointed out that the kinematics estimator was trained with the arm held at a specific orientation. Therefore, a drop in performance is expected in case of multiposition use, as it normally happens in daily life movements. This problem may be mitigated collecting training data in multiple positions [26].

\section{B. Mirror Training}

The fact that ANNs could be successfully trained to provide hand kinematic estimation from the EMG of the ipsilateral forearm shows that the information embedded in the surface EMG of the forearm is sufficient to decode the user intent, at least when limited to the investigated movements. However, ipsilateral kinematics is not available in amputees. The proposed training strategy, which requires the kinematics from the limb contralateral to the one used to gather EMG, can be directly replicated in unilateral amputees. Another possibility would be to ask the amputee to imitate the movements of a human teacher with his phantom limb, as in [27], and to use the teacher kinematics as target for the ANNs. The teacher imitation modality has the advantage of being applicable also in bilateral amputees. However, mirror training may be a preferable option in case of unilateral amputees because synchrony between the normal and the phantom limb of the amputee may be easier to achieve than synchrony between the teacher movement and the amputee imitation. A temporal misalignment between input and target would affect the ANN training procedure.

The study was conducted on normally-limbed subjects to test the feasibility of the approach and to provide a benchmark for future validation in amputees, as it is conventionally done in the myoelectric control research community $[7,16,17,28]$. 
A

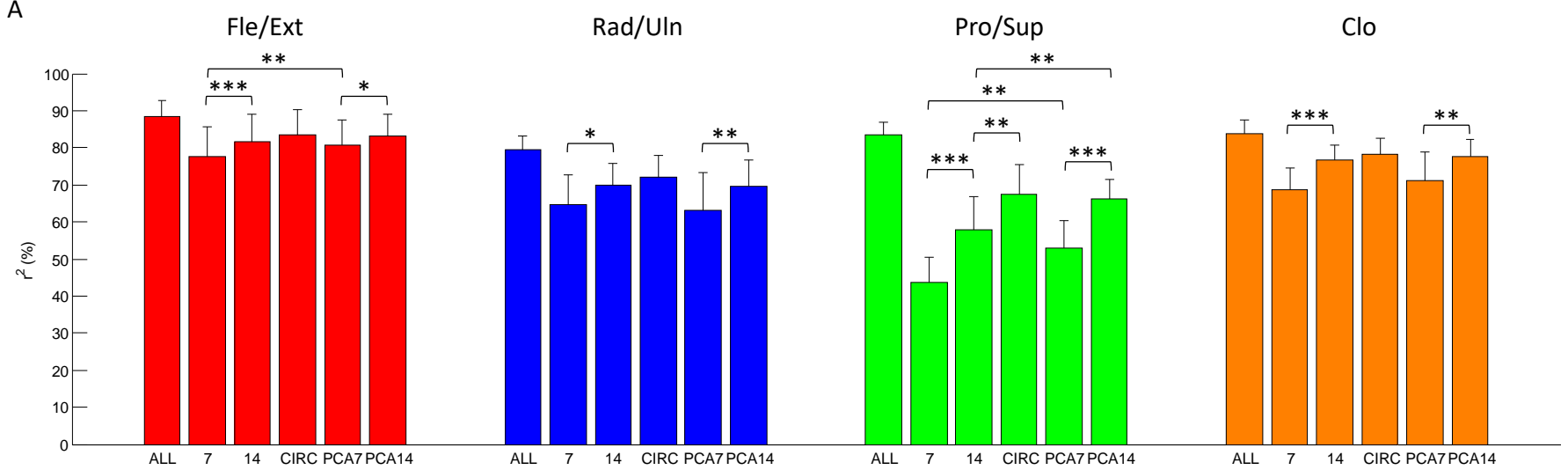

B

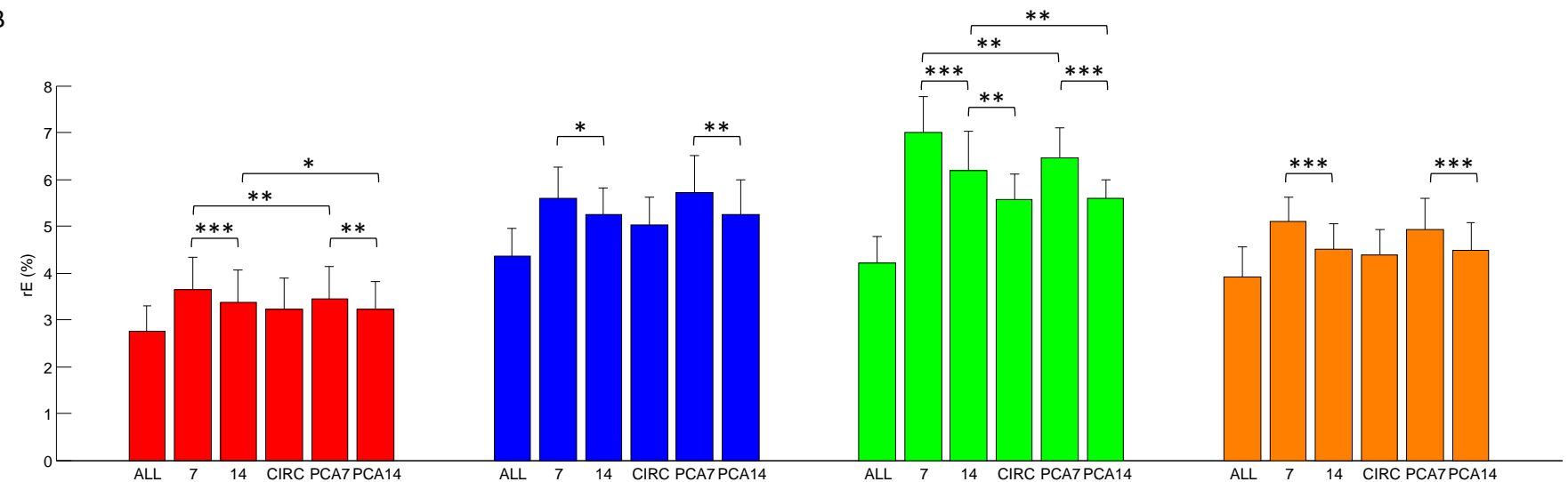

Fig. 4. Mean \pm standard deviation of the coefficient of determination (A) and mean relative error (B) between the measured and estimated angular position of the contralateral hand using the different feature sets. ALL: all the channels; 7, 14 and CIRC: 7, 14 and 26 channels resulting from feature selection (Fig. 2 A, B and C); PCA7 (PCA14): the first 7 (14) principal components; * $\mathrm{P}<0.05$, ** $\mathrm{P}<0.01$, *** $\mathrm{P}<0.001$.

Nevertheless, it has been shown that amputees can voluntarily produce different movements of their phantom hand and that these movements are associated with stump muscle activity patterns distinct and reproducible over time [29]. The fact that distinguishable and consistent patterns in the surface EMG can be associated to diverse movements also in amputees provides the physiological basis to make the proposed training strategy potentially applicable in prosthesis users.

\section{High-density EMG}

The investigation on feature projection/selection indicated the benefits of employing a high-density configuration in collecting the EMG, at least when only the envelopes of the signal are used as features. Indeed, using all channels, all the angles could be estimated with $r^{2}>79 \%$. When the feature set dimensionality was reduced, the estimation of all angles was negatively affected, especially for pronation/supination $\left(r^{2} \approx\right.$ $43 \%$, using 7 channels only). Flexion/extension could still be estimated with a $r^{2} \approx 77 \%$, even with the minimal configuration. This was somehow expected from the forearm anatomy. Indeed, many of the muscles involved in flexion/extension (flexor carpi ulnaris, flexor carpi radialis, flexor digitorum superficialis, extensor carpi radialis, extensor digitorum, extensor digiti minimi, extensor carpi ulnaris) are superficial. Therefore, even an equispaced placement of 7 electrodes around the forearm circumference provides a good spatial sampling of their surface activity, although less comprehensive that the one available from the matrixes. On the other hand, the supinator is a deep muscle, thus its activity is relatively weaker at the surface of the skin. Pronation is mainly served by two muscles: pronator teres and pronator quadratus. However, being the latter located in the distal forearm, transradial amputees only retain the pronator teres. The situation is made even more complex by the fact that the pronator teres is partly covered by the bicipital aponeurosis, which reduces the signal amplitude at the surface of the skin. Nevertheless, high-density EMG enables to cope with the fact that EMG produced by the muscle responsible for pronation/supination are low and/or buried by cross-talk from other muscles.

The abundance of channels results in surface EMG patterns which are distinguishable and consistent over time even in case of pronation/supination, although of low amplitude. Moreover, in dynamic conditions, the relative sliding of the muscles beneath the skin can move them away from the electrode detection areas while the configuration that we proposed covers the full forearm circumference with several closely spaced electrodes. Nevertheless, it is difficult to 
predict to what extent the muscle sliding problem may be relevant in amputees. During surgical amputation, the residual muscle or tendon are directly sutured to the bone (myodesis) to provide stability. Therefore, it is expected that changes in muscle shape and length are less pronounced in amputees than in normally-limbed subjects.

\section{Feature Reduction}

A configuration with 14 electrodes equispaced around the forearm resulted in better performance than 7 electrodes but an additional inclusion of 12 channels (array like configuration) caused a significant further increase in the estimation quality only for pronation/supination. In general, the full high-density recordings outperformed all the investigated channel subsets. However, the arrangement with 26 channels retained reasonable performance, especially for three DOFs $\left(r^{2}\right.$ within the range $\approx 67 \%$ (pronation/supination) - $83 \%$ (flexion/extension), $r E<6 \%$ ).

Seven components explained $\sim 92 \%$ of the multi-channel EMG signal variance. Nevertheless, the extra information carried by the subsequent seven components was relevant for the decoding of the four DOFs. The number of channels being equal, channel projection sometimes resulted superior to channel selection, maybe because it provides more comprehensive information.

Although the performance needed to be quantified with metrics, indices often reported to describe the accuracy of myoelectric control systems do not necessarily relate to the usability of the system [15]. Therefore, it is unclear if the gain in performance when using the full electrode system configuration is functionally relevant. From Fig. 4, the effective benefit from high-density recording concerns mainly the pronation/supination DOF.

\section{E. Implementation}

Most studies on myoelectric control make use of 8 or less electrodes $[4,7,8,10,11]$, mainly for practical reasons, i.e. to keep the computational time low and to simplify positioning. Regarding the processing time, it must be taken into account that some signal processing frameworks compensate for the reduced number of channels increasing the number of feature extracted from them [30], while the scheme that we proposed simply relies on the signal envelope [27, 31]. In addition, considering the sampling rate of our data and that filters of the $2^{\text {nd }}$ order were used, the delay introduced by the estimation is only $\sim 100 \mathrm{~ms}$, in line with real-time requirements of myoelectric control [32]. Moreover, the electrode placement time is limited because there is no need to target specific muscles $[7,27]$ as it is sometimes done in setups that make use of a limited number of channels [8]. Electrode technology may in the near future further simplify positioning (e.g., with textile electrode systems [33]). However, it should be noted that the present study also shows that the number of electrodes can be reduced from the full configuration proposed by retaining acceptable performance. This is important because an increase in the electrode number would imply an increase in power consumption and cost of the prosthesis.
To actuate the proposed training strategy in a clinical setting, kinematics may be measured using a simpler system than the one applied in this study, consisting e.g. of goniometers and torsiometers. Furthermore, the protocol that we used for training/validation includes many contractions because the study was meant to analyze the system performance in a systematic fashion. As far as the training protocol includes movements representative of the DOFs at the joints of interest, the MLPs should be able to capture the association between EMG and kinematics. Therefore, to speed up the training procedure in a clinical setting, the protocol could include only free movements in 3D. This would also allow evaluating the performance of the system in case of transition from a DOF to another without returning to the resting state.

\section{CONCLUSION}

We have shown that kinematics from the complex wristhand can be estimated from high-density EMG signals of the contralateral arm while the subject performs mirrored movements involving the simultaneous articulation of four DOFs. When reducing the number of channels used for the estimation, the system retained good performance for at least three DOFs. The approach proposed in this paper represents a potential solution that can be practically applied in unilateral transradial amputees for simultaneous and proportional control of multiple DOFs.

\section{ACKNOWLEDGMENT}

The authors would like to thank Dr. Ning Jiang for helpful discussion.

\section{References}

[1] M. C. Carrozza, P. Dario, F. Vecchi, S. Roccella, M. Zecca and F. Sebastiani, "The CyberHand: On the design of a cybernetic prosthetic hand intended to be interfaced to the peripheral nervous system," in IEEE International Conference on Intelligent Robots and Systems, 2003, pp. 26422647.

[2] The i-LIMB Hand http://www.touchbionics.com/i-LIMB

\section{[3] Otto Bock http://www.ottobock.com}

[4] M. F. Lucas, A. Gaufriau, S. Pascual, C. Doncarli and D. Farina, "Multichannel surface EMG classification using support vector machines and signalbased wavelet optimization," Biomedical Signal Processing and Control, vol. 3, pp. 169-174, 2008.

[5] L. J. Hargrove, K. Englehart and B. Hudgins, "A comparison of surface and intramuscular myoelectric signal classification," IEEE Transactions on Biomedical Engineering, vol. 54, pp. 847-853, 2007.

[6] D. H. Silcox 3rd, M. D. Rooks, R. R. Vogel and L. L. Fleming, "Myoelectric prostheses. A long-term follow-up and a study of the use of alternate prostheses," J. Bone Joint Surg. Am., vol. 75, pp. 1781-1789, Dec, 1993.

[7] N. Jiang, K. B. Englehart and P. A. Parker, "Extracting simultaneous and proportional neural control information for multiple-dof prostheses from the 
surface electromyographic signal," IEEE Transactions on Biomedical Engineering, vol. 56, pp. 1070-1080, 2009.

[8] J. L. G. Nielsen, S. Holmgaard, N. Jiang, K. B. Englehart, D. Farina and P. A. Parker, "Simultaneous and proportional force estimation for multifunction myoelectric prostheses using mirrored bilateral training," IEEE Transactions on Biomedical Engineering, vol. 58, pp. 681-688, 2011.

[9] R. Merletti, A. Holobar and D. Farina, "Analysis of motor units with highdensity surface electromyography," Journal of Electromyography and Kinesiology, vol. 18, pp. 879-890, 2008.

[10] K. Englehart and B. Hudgins, "A robust, real-time control scheme for multifunction myoelectric control," IEEE Trans. Biomed. Eng., vol. 50, pp. 848-854, Jul, 2003.

[11] J. L. G. Nielsen, S. Holmgaard, N. Jiang, K. Englehart, D. Farina and P. Parker, "Enhanced EMG signal processing for simultaneous and proportional myoelectric control," in Proceedings of the 31st Annual International Conference of the IEEE Engineering in Medicine and Biology Society: Engineering the Future of Biomedicine, EMBC 2009, 2009, pp. 4335-4338.

[12] A. Gilroy, B. MacPherson and L. Ross, Atlas of Anatomy. Thieme, 2008.

[13] F. Sebelius, L. Eriksson, C. Balkenius and T. Laurell, "Myoelectric control of a computer animated hand: A new concept based on the combined use of a tree-structured artificial neural network and a data glove," Journal of Medical Engineering and Technology, vol. 30, pp. 2-10, 2006.

[14] C. L. Pulliam, J. M. Lambrecht and R. F. Kirsch, "Electromyogram-based neural network control of transhumeral prostheses," Journal of Rehabilitation Research and Development, vol. 48, pp. 739, 2011.

[15] L. Hargrove, Y. Losier, B. Lock, K. Englehart and B. Hudgins, "A realtime pattern recognition based myoelectric control usability study implemented in a virtual environment," Conf. Proc. IEEE Eng. Med. Biol. Soc., vol. 2007, pp. 4842-4845, 2007.

[16] L. J. Hargrove, E. J. Scheme, K. B. Englehart and B. S. Hudgins, "Multiple Binary Classifications via Linear Discriminant Analysis for Improved Controllability of a Powered Prosthesis," Neural Systems and Rehabilitation Engineering, IEEE Transactions on, vol. 18, pp. 49-57, 2010.

[17] L. H. Smith, L. J. Hargrove, B. A. Lock and T. A. Kuiken, "Determining the Optimal Window Length for Pattern Recognition-Based Myoelectric Control: Balancing the Competing Effects of Classification Error and Controller Delay," Neural Systems and Rehabilitation Engineering, IEEE Transactions on, vol. 19, pp. 186-192, 2011.

[18] S. Muceli, N. Jiang and D. Farina, "Multichannel surface EMG based estimation of bilateral hand kinematics during movements at multiple degrees of freedom," Conf. Proc. IEEE Eng. Med. Biol. Soc., vol. 2010, pp. 6066$6069,2010$.

[19] H. Hermens, B. Freriks, R. Merletti, D. F. Stegeman, J. Blok, C. Rau, C. Disselhorst-Klug and G. Hagg, "European Recommendations for Surface Electromyography. Results of the SENIAM project," Roessingh Research and Development, 1999.

[20] R. Merletti and P. A. Parker, Electromyography: Physiology, Engineering, and Noninvasive Applications. Wiley-IEEE Press, 2004.

[21] K. Levenberg, "A Method for the Solution of Certain Non-Linear Problems in Least Squares," The Quarterly of Applied Mathematics, vol. 2, pp. 164-168, 1944.

[22] D. A. Winter, Biomechanics and Motor Control of Human Movement. Wiley-Interscience, 1990.

[23] A. d'Avella, A. Portone, L. Fernandez and F. Lacquaniti, "Control of fastreaching movements by muscle synergy combinations," J. Neurosci., vol. 26, pp. 7791-7810, Jul 26, 2006.
[24] H. Hotelling, "Analysis of a complex of statistical variables into principal components," J. Educ. Psychol., vol. 24, pp. 498-520, 1933.

[25] E. J. Scheme, K. B. Englehart and B. S. Hudgins, "Selective classification for improved robustness of myoelectric control under nonideal conditions," IEEE Trans. Biomed. Eng., vol. 58, pp. 1698-1705, Jun, 2011.

[26] E. Scheme, A. Fougner, Ø. Stavdahl, A. Chan and K. Englehart, "Examining the adverse effects of limb position on pattern recognition based myoelectric control," in Engineering in Medicine and Biology Society (EMBC), 2010 Annual International Conference of the IEEE, 2010, pp. 63376340 .

[27] C. Castellini, E. Gruppioni, A. Davalli and G. Sandini, "Fine detection of grasp force and posture by amputees via surface electromyography," Journal of Physiology-Paris, vol. 103, pp. 255-262, 2009.

[28] A. Young, L. Hargrove and T. Kuiken, "The Effects of Electrode Size and Orientation on the Sensitivity of Myoelectric Pattern Recognition Systems to Electrode Shift," IEEE Trans. Biomed. Eng., Jun 9, 2011.

[29] K. T. Reilly, C. Mercier, M. H. Schieber and A. Sirigu, "Persistent hand motor commands in the amputees' brain," Brain, vol. 129, pp. 2211-2223, Aug, 2006.

[30] M. Zecca, S. Micera, M. C. Carrozza and P. Dario, "Control of multifunctional prosthetic hands by processing the electromyographic signal," Crit. Rev. Biomed. Eng., vol. 30, pp. 459-485, 2002.

[31] C. Castellini and P. van der Smagt, "Surface EMG in advanced hand prosthetics," Biol. Cybern., vol. 100, pp. 35-47, 2009.

[32] T. R. Farrell and R. F. Weir, "The optimal controller delay for myoelectric prostheses," IEEE Transactions on Neural Systems and Rehabilitation Engineering, vol. 15, pp. 111-118, 2007.

[33] D. Farina, T. Lorrain, F. Negro and N. Jiang, "High-density EMG Etextile systems for the control of active prostheses," in Engineering in Medicine and Biology Society (EMBC), 2010 Annual International Conference of the IEEE, 2010, pp. 3591-3593. 